\title{
REMARKS ON FUNDAMENTAL GROUPS OF COMPLEMENTS OF DIVISORS ON ALGEBRAIC VARIETIES
}

\author{
BY ICHIRO SHIMADA
}

\section{Introduction}

We work over the complex number field $\boldsymbol{C}$, and consider the topological fundamental group of the complement of a divisor on a nonsingular projective variety.

Let $V$ be a nonsingular connected projective variety of dimension $\geqq 2$ and let $D \subset V$ be a reduced divisor. We denote by $p: L \rightarrow V$ the line bundle corresponding to the invertible sheaf $\mathcal{O}_{V}(D)$, and we put

$$
L^{\times}:=L \backslash\{\text { the zero section }\} .
$$

We fix base points $b \in V \backslash D$ and $b^{\prime} \in L^{\times}$such that $p\left(b^{\prime}\right)=b$. There exists a unique section $s: V \rightarrow L$ which defines $D$ and passes through $b^{\prime}$. By restricting $s$ to $V \backslash D$, we get a morphism $V \backslash D \rightarrow L^{\times}$, which we denote by the same letter s. We consider the homomorphism

$$
s_{*}: \pi_{1}(V \backslash D, b) \longrightarrow \pi_{1}\left(L^{\times}, b^{\prime}\right) .
$$

In various "good" situations (for example, when $D$ is very ample and nonsingular), this homomorphism is an isomorphism. The following is a special case of Nori's result [3, Corollary 2.10], which is one of the corollaries of his Weak Lefschetz Theorem.

Proposition (NORI). Suppose that $D$ is irreducible and not composed of a pencil. If the singular locus of $D$ is of codimension $\geqq 3$ in $V$, then $\pi_{1}(V \backslash D)$ is isomorphic to $\pi_{1}\left(L^{\times}\right)$.

In this paper, we give another condition under which $s_{*}$ is an isomorphism, using some ideas originated in [4]. As an application, we compute the fundamental groups of complements of certain singular plane curves.

Let $V$ be as above and let $\delta$ be a linear system on $V$. We put

$$
\text { Bs } \downarrow:=\{x \in V ; x \in D \text { for all } D \in \oslash\} \text {. }
$$

We also put $V^{0}:=V \backslash$ Bs $\delta$. Then there is a morphism $\Phi: V^{0} \rightarrow \delta^{2}$ induced by $\emptyset$

Received September 13, 1993 ; revised April 19, 1994. 
where $\delta^{2}$ is the dual projective space of $\delta$. As above, we denote by $p: L \rightarrow V$ the line bundle corresponding to $\mathcal{O}_{V}(D)$ where $D$ is an arbitrary member of $b$, and by $L^{\times}$the complement of the zero section of $L$. We also put

$$
\grave{\delta}_{n r}:=\{D \in \delta ; D \text { is not reduced }\} \text {. }
$$

The main result is as follows:

Proposition 1. Suppose that D has no fixed components and the image of $\Phi$ is of dimension $\geqq 2$. Suppose also one of the following holds; (i) $\grave{b}_{n r} \subset \mathcal{D}$ is of codimension $\geqq 2$, or (ii) every fiber of $\Phi$ is of codimension $\geqq 2$ in $V^{0}$. Then, for a general member $D \in \mathfrak{D}, s_{*}$ is an isomorphism.

Note that if $s_{*}$ is an isomorphism, we have a commutative diagram

$$
\begin{gathered}
\pi_{1}(V \backslash D) \cong \pi_{1}\left(L^{\times}\right) \\
i_{*} \searrow p_{*} \\
\pi_{1}(V),
\end{gathered}
$$

where $i: V \backslash D \subset V$ is the inclusion. By this commutative diagram, we have an exact sequence

$$
\longrightarrow \pi_{2}(V) \stackrel{\partial}{\longrightarrow} Z \longrightarrow \pi_{1}(V \backslash D) \longrightarrow \pi_{1}(V) \longrightarrow 1,
$$

derived from the homotopy exact sequence of $L^{\times} \rightarrow V$. It is easy to see that the image of $Z \rightarrow \pi_{1}(V \backslash D)$ is contained in the center of $\pi_{1}(V \backslash D)$. Thus $\pi_{1}(V \backslash D)$ is a central extension of $\pi_{1}(V)$ by a cyclic group.

We shall study the boundary homomorphism $\partial$ in the sequence $(0.2)$. The homology class $[D] \in H_{2 n-2}(V, \boldsymbol{Z})$ of the divisor $D$, where $n=\operatorname{dim} V$, defines a linear form

$$
\delta: H_{2}(V, \boldsymbol{Z}) \longrightarrow \boldsymbol{Z}
$$

by the intersection paring $H_{2}(V, \boldsymbol{Z}) \times H_{2 n-2}(V, \boldsymbol{Z}) \rightarrow \boldsymbol{Z}$.

Proposition 2. Suppose that $s_{*}$ is an isomorphism. Then the boundary map $\partial$ in (0.2) is given by

$$
\pi_{2}(V) \stackrel{\eta}{\longrightarrow} H_{2}(V, \boldsymbol{Z}) \stackrel{\delta}{\longrightarrow} \boldsymbol{Z},
$$

where $\eta$ is the Hurewicz map and $\delta$ is the linear form defined above.

Let $\boldsymbol{Z}_{\geq 0}$ be the set of non-negative integers. We put

$$
\mathcal{S}_{d}:=\left\{(i, j, k) \in\left(\boldsymbol{Z}_{\geq 0}\right)^{3} ; i+j+k=d\right\} .
$$

For a subset $S \subset \mathcal{S}_{d}$ of $\mathcal{S}_{d}$, we denote by $\mathfrak{d}(S)$ the linear system of all curves on $\boldsymbol{P}^{2}$ whose defining equations are of the form 


$$
\sum_{(2, \jmath, k) \in S} a_{\imath j k} X_{0}^{i} X_{1}^{j} X_{2}^{k}=0
$$

where $\left(X_{0}: X_{1}: X_{2}\right)$ are homogeneous coordinates of $\boldsymbol{P}^{2}$. As a corollary of Propositions 1 and 2, we have the following:

Proposition 3. Suppose that $\operatorname{Card}(S \cap\{i=0\}) \geqq 2, \operatorname{Card}(S \cap\{\jmath=0\}) \geqq 2$, and Card $(S \cap\{k=0\}) \geqq 2$. Let $D$ be a general member of $\mathrm{D}(S)$. Then the fundamental group $\pi_{1}\left(\boldsymbol{P}^{2} \backslash D\right)$ is isomorphic to the cyclic group of order $d$.

Example 1. We fix three points $P_{1}=(1: 0: 0), P_{2}=(0: 1: 0)$ and $P_{3}=(0: 0: 1)$ on $\boldsymbol{P}^{2}$. Let $m_{1}, m_{2}, m_{3}$ and $d$ be non-negative integers. Let $\delta$ be the linear system of all curves of degree $d$ in $\boldsymbol{P}^{2}$ which have singularity of multiplicity $m_{\imath}$ at each point $P_{\imath}$ for $\imath=1,2,3$. Suppose that $m_{1}+m_{2}<d, m_{2}+m_{3}<d$, and $m_{3}+m_{1}<d$. Then the fundamental group of the complement of a general member of $b$ is isomorphic to $Z /(d)$.

Example 2 (cf. [1, Chapter 4 (3.11)]). We fix affine coordinates $(x, y)$ on $\boldsymbol{P}^{2}$. Let $d_{1}>d_{2}>\cdots>d_{\mu}$ be a decreasing sequence of positive integers with $\mu \geqq 2$. Consider the projective plane curve $C$ defined by an inhomogeneous equation

$$
f_{d_{\mu}}(x, y)+\cdots+f_{d_{2}}(x, y)+f_{d_{1}}(x, y)=0,
$$

where $f_{d_{i}}(x, y)(i=1, \cdots, \mu)$ are general homogeneous polynomials of degree $d_{\imath}$. Then $\pi_{1}\left(\boldsymbol{P}^{2} \backslash C\right)$ is isomorphic to the cyclic group of order $d_{1}$.

In the last section, we give some other elementary examples.

\section{Proof of Proposition 1}

First, we shall show, by contradiction, that the assumption (ii) implies the assumption (i). Suppose that there exists an irreducible component $\mathfrak{b}^{\prime}$ of $\grave{b}_{n r}$ of dimension $\operatorname{dim} \delta-1$. Let $\Lambda(\delta) \subset H^{\circ}(V, L)$ be the linear subspace corresponding to $\delta$, and let $C\left(\delta^{\prime}\right) \subset \Lambda(\delta)$ be the cone over $\delta^{\prime}$. Let $s_{0} \in C\left(\delta^{\prime}\right)$ be a general point. We may assume that $C\left(\delta^{\prime}\right)$ is nonsingular at $s_{0}$. Then the tangent space $T_{s_{0}, C\left(\delta^{\prime}\right)}$ to $C\left(\delta^{\prime}\right)$ at $s_{0}$ is canonically isomorphic to a linear subspace $\Lambda^{\prime}$ of codimension 1 in $\Lambda(\delta)$. Let $M$ be a small coordinate neighborhood of $s_{0}$ in $C\left(\boldsymbol{D}^{\prime}\right)$, and let

$$
\phi: \Delta:=\left\{z \in C^{\operatorname{dim} c\left(\delta^{\prime}\right)} ;|z|<1\right\} \stackrel{\sim}{\longrightarrow} M
$$

be the coordinates. We denote by $s_{z}$ the global section of $L$ corresponding to $\phi(z) \in C\left(\mathrm{~b}^{\prime}\right)$, and by $D_{z}$ the divisor defined by $s_{z}=0$. Since $s_{0} \in C\left({\mathfrak{D}^{\prime}}^{\prime}\right)$ is general and $M$ is small, there exist analytic families of divisors $\left\{E_{z}\right\}_{z \in \Delta}$ and $\left\{F_{z}\right\}_{z \in \Delta}$ over $\Delta$ such that

$$
D_{z}=l \cdot E_{z}+F_{z} \quad(l \geqq 2),
$$


and $E_{z}$ are reduced irreducible divisors for all $z$. Let $U \subset V$ be a classically open neighborhood of $V$ around a general point of $E_{0}$, over which there exists a trivialization

$$
\left.L\right|_{U} \cong \boldsymbol{C} \times U
$$

of the line bundle $L$. Then there exist families of defining functions $\left\{t_{2}\right\}$ and $\left\{u_{z}\right\}$ of $E_{z}$ and $F_{z}$, respectively, on $U$ such that

$$
s_{z}=t_{z}^{l} \cdot u_{z}
$$

holds on $U$, where we consider $\left.s_{z}\right|_{U}$ as a function on $U$ by the above trivialization. Let $s^{\prime} \in T_{s_{0}, C\left(\delta^{\prime}\right)}$ be an arbitrary tangent vector to the cone $C\left(\delta^{\prime}\right)$ at $s_{0}$. Then we can deform (1.0) to the direction $s^{\prime}$ in the first order. Let $\varepsilon$ be a dual number; $\varepsilon^{2}=0$. We write the first two terms of expansions of $s_{z}, t_{z}$ and $u_{z}$ of this deformation as follows;

$$
s_{\varepsilon}=s_{0}+\varepsilon s^{\prime}, \quad t_{\varepsilon}=t_{0}+\varepsilon t^{\prime}, \text { and } u_{\varepsilon}=u_{0}+\varepsilon u^{\prime} .
$$

Then, considering $s^{\prime}$ as an element of $\Lambda(\S)$ by the canonical isomorphism $T_{s_{0}, C\left(\delta^{\prime}\right)} \cong \Lambda^{\prime} \subset \Lambda(\emptyset)$ and regarding $\left.s^{\prime}\right|_{U}$ as a function as above, we see that

$$
s^{\prime}=t_{0}^{l-1}\left(l t^{\prime} u_{0}+t_{0} u^{\prime}\right)
$$

holds on $U$. Thus, locally on $U$, the divisor $\left\{s^{\prime}=0\right\}$ contains $E_{0}$ with multiplicity $\geqq l-1>0$. Since $E_{0}$ is irreducible, this implies that the divisor $\left\{s^{\prime}=0\right\}$ contains $E_{0}$ globally. Since $T_{s_{0}, C\left(D^{\prime}\right)} \cong \Lambda^{\prime} \subset \Lambda(\delta)$ is a linear subspace of codimension 1 and $s^{\prime} \in T_{s_{0}, C\left(b^{\prime}\right)}$ is arbitrary, this means that $E_{0} \cap V^{0}$ is mapped to a point by the morphism $\Phi$. (Note that since $\delta$ has no fixed components by the assumption, Bs $d$ is of codimension $\geqq 2$ in $V$. Thus $E_{0} \cap V^{0}$ is non-empty.) This contradicts the assumption (ii). Therefore we may and will assume the assumption (i) from the outset.

Let $q_{0}$ be a general point of $\delta^{2}$. Since $\operatorname{dim} \Phi\left(V^{0}\right) \geqq 2$, the inverse image $\Phi^{-1}\left(q_{0}\right) \subset V^{0}$ is either empty or of codimension $\geqq 2$. We put $V^{1}:=V^{0} \backslash \Phi^{-1}\left(q_{0}\right)$. Let $A$ be the space of all hyperplanes $H$ in the projective space $\delta^{2}$ such that $H \not \supset q_{0}$. Then $A$ is isomorphic to an affine space. We put

$$
W:=\left\{(y, H) \in V^{1} \times A ; \Phi(y) \in H\right\}
$$

and $U:=\left(V^{1} \times A\right) \backslash W$. Then $W$ is a Zariski closed subset of codimension 1 in $V^{1} \times A$. We give $W$ the reduced structure. For $H \in A$, we denote by $W_{H}$ the scheme theoretic intersection $W \cap\left(V^{1} \times\{H\}\right)$, which is regarded as a divisor of $V^{1}$. Then we have $W_{H}=D_{H} \cap V^{1}$, where $D_{H}$ is the divisor of $V$ corresponding to $H \in\left(\delta^{2}\right)^{2}=\varnothing$. Since Bs $\delta$ is of codimension $\geqq 2$ in $V, V^{1}$ admits a non-singular projective compactification $V$ such that $V \backslash V^{1}=\Phi^{-1}\left(q_{0}\right) \cup B s \delta$ is of codimension $\geqq 2$. Combining this with the assumption (i), we can use [4, Theorem 1$]$ and get isomorphisms 


$$
\pi_{1}(\mathcal{U}) \cong \pi_{1}\left(V^{1} \backslash W_{H}\right) \cong \pi_{1}\left(V \backslash D_{H}\right)
$$

for a general $H \in A$. These isomorphisms are induced by the inclusions $V^{1} \backslash W_{H}$ $\hookrightarrow \mathcal{Q}$ and $V^{1} \backslash W_{H} \subset V \backslash D_{H}$.

For $y \in V^{1}$, let $l_{y}$ be the line in $\delta^{2}$ connecting $\Phi(y)$ and $q_{0}$. Note that since $\Phi(y) \div q_{7}$, the line $l_{y}$ is uniquely determined. Since $\Phi * \mathcal{O}_{\triangleright \sim}(1)=\mathcal{O}_{V 0}\left(D_{H} \cap V^{0}\right)$, we have isomorphisms which fit into the commutative diagram;

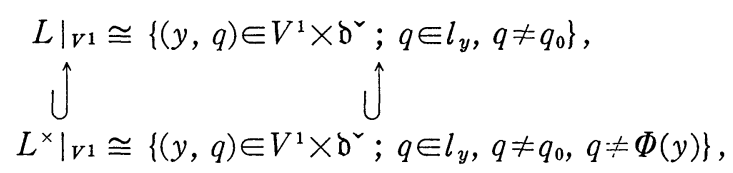

and are compatible with the projections to $V^{1}$. Under these isomorphisms, the section $s\left(D_{H}\right)$ of $L \rightarrow V$ defining $D_{H}$ is given by

$$
y \longmapsto\left(y, H \cap l_{y}\right)
$$

over $V_{1}$. (Note that the above isomorphisms are unique up to the automorphisms of the fiber bundles $p: L \rightarrow V$ and $p: L^{\times} \rightarrow V$ by the group $\boldsymbol{C}^{\times}$acting on fibers by the scalar multiplication. For the section $y \mapsto\left(y, H \cap l_{y}\right)$ to pass through a given point $b^{\prime} \in L^{\times}$as in Introduction, we have to choose the isomorphisms in a suitable way.) If $(y, H) \in \mathcal{U}$, then $H$ intersects the line $l_{y}$ at a point on $l_{y} \backslash\left\{\Phi(y), q_{0}\right\}$, because $y \notin D_{H}$. Therefore, using the above isomorphisms, we have a morphism

$$
\begin{aligned}
U & \left.\longrightarrow L^{\times}\right|_{V 1} \\
(y, H) & \longmapsto\left(y, H \cap l_{y}\right) \quad\left(=s\left(D_{H}\right)(y)\right) .
\end{aligned}
$$

This makes $U$ a locally trivial fiber space over $\left.L^{\times}\right|_{V 1}$, whose fiber is the space of all hyperplanes of $\delta^{2}$ passing through a given point $\left(\neq q_{0}\right)$ and not containing $q_{0}$, which is isomorphic to an affine space. Hence we get isomorphisms

$$
\pi_{1}(U) \cong \pi_{1}\left(\left.L^{\times}\right|_{V 1}\right) \cong \pi_{1}\left(L^{\times}\right) .
$$

(Note that $L^{\times} \backslash\left(\left.L^{\times}\right|_{V 1}\right)$ is of codimension $\geqq 2$.) Combining this with (1.1), we have

$$
\pi_{1}\left(V \backslash D_{H}\right) \cong \pi_{1}\left(L^{\times}\right)
$$

for a general $H \in A$. By the constructions, this isomorphism is induced by the section $s\left(D_{H}\right): V \backslash D_{H} \rightarrow L^{\times}$.

\section{Proof of Proposition 2}

We fix base points $b$ of $V \backslash D$, and $*$ of an oriented 2 -sphere $S^{2}$. Let

$$
g:\left(S^{2}, *\right) \longrightarrow(V, b)
$$


be a continuous map. We shall consider the image of the homotopy equivalence class $[g] \in \pi_{2}\left(V, b_{0}\right)$ via the boundary map $\partial$ in $(0.2)$. Deforming $g$ homotopically relative to $*$, we may assume that the image of $g$ intersects the divisor $D$ at its nonsingular points transversely, and $g^{-1}\left(g\left(S^{2}\right) \cap D\right)$ is a finite set of points. We put

$$
g^{-1}\left(g\left(S^{2}\right) \cap D\right)=\left\{P_{1}, \cdots, P_{k}\right\} .
$$

Let $\mathcal{S} \rightarrow V$ be the oriented $S^{1}$-bundle associated with $L^{\times} \rightarrow V$, where the orientation is induced by the complex structure of the fibers of $L^{\times} \rightarrow V$. The section $s=s(D): V \backslash D \rightarrow L^{\times}$induces a section $r(D)$ of $\left.\mathcal{S}\right|_{V \backslash D \rightarrow V} \backslash D$. By pulling back, we get a section of $g^{*} \mathcal{S} \rightarrow S^{2}$ over $S^{2} \backslash\left\{P_{1}, \cdots, P_{k}\right\}$, which we shall denote by $r^{\prime}(D)$.

For simplicity, we fix some notation. Let $P$ be a point of $S^{2}$, and let $\Delta \subset$ $S^{2}$ be a subset which contains $P$ in its interior and is homeomorphic to the closed disk $\{z \in C ;|z| \leqq 1\}$. We denote by $S_{P}^{1}$ the fiber of $g^{*} \mathcal{S} \rightarrow S^{2}$ over $P$, which is oriented. There exists a trivialization

$$
\left.g * \mathcal{S}\right|_{\Delta} \cong S_{P}^{1} \times \Delta,
$$

which is the identity on $S_{P}^{1}$. Now suppose that there is a section $\sigma: \partial \Delta \rightarrow g^{*} \mathcal{S}$ of $g^{*} \mathcal{S} \rightarrow S^{2}$ over $\partial \Delta$. We define an integer $m(\sigma, \Delta, P)$ to be the mapping degree of the composition

$$
\left.\partial \Delta \stackrel{\sigma}{\longrightarrow} g^{*} \mathcal{S}\right|_{\Delta} \cong S_{P}^{1} \times \Delta \stackrel{\mathrm{pr}_{1}}{\longrightarrow} S_{P}^{1},
$$

where the orientation of $\partial \Delta$ is induced from that of $S^{2}$. It is easy to see that this number $m(\sigma, \Delta, P)$ is independent of the choice of the trivialization of $\left.g^{*} \mathcal{S}\right|_{\Delta}$. If the section $\sigma$ extends over the whole $\Delta$, then $m(\sigma, \Delta, P)=0$. By the definition of the boundary map $\partial$, if $\Delta$ contains the base point $*$ in its interior and the section $\sigma$ is defined over the complement $S^{2} \backslash$ (the interior of $\Delta$ ), then

$$
m(\sigma, \Delta, *)=\partial([g]) .
$$

We fix a small closed disk $\Delta_{\imath}$ on $S^{2}$ with the center $P_{\imath}$. By the definition of the section $r^{\prime}(D)$, we have

(2.2) $m\left(r^{\prime}(D), \Delta_{i}, P_{\imath}\right)=$ the local intersection number of $g\left(S^{2}\right)$ and $D$ at $P_{\imath}$.

(Since $g\left(S^{2}\right)$ and $D$ intersect transversely, these numbers are \pm 1 .)

Let $\omega_{i}: I \rightarrow S^{2}$ be a path from $*$ to a point $e_{i} \in \partial \Delta_{\imath}$ such that each $\omega_{i}$ is injective, $\omega_{i}(I) \cap \Delta_{\imath}=\left\{e_{\imath}\right\}$, and if $\imath \neq j$, then $\omega_{i}(I) \cap \Delta_{\jmath}=\emptyset$ and $\omega_{i}(I) \cap \omega_{j}(I)=\{*\}$. We put

and

$$
T_{\imath}:=\Delta_{i} \cup \omega_{i}(I),
$$

$$
T:=\bigcup_{\imath=1}^{k} T_{\imath} \text {. }
$$

Let $d$ be a standard distance on $S^{2}$. For a small positive real number $\varepsilon>0$, we put 


$$
\begin{aligned}
T_{\imath, \varepsilon} & :=\left\{P \in S^{2} ; \min _{Q \in T_{\imath}} d(P, Q) \leqq \varepsilon\right\}, \\
T_{\varepsilon} & :=\left\{P \in S^{2} ; \min _{Q \in T} d(P, Q) \leqq \varepsilon\right\} .
\end{aligned}
$$

Then both of $T_{\varepsilon}$ and $T_{\imath, \varepsilon}$ contain $*$ in their interiors and, if $\varepsilon$ is small enough, they are homeomorphic to the closed disk. Moreover, since the section $r^{\prime}(D)$ is defined over $S^{2} \backslash$ (the interior of $T_{\varepsilon}$ ), we have, from (2.1), that

$$
m\left(r^{\prime}(D), T_{\varepsilon}, *\right)=\partial([g]) .
$$

On the other hand, it is obvious that $m\left(r^{\prime}(D), \Delta_{i}, P_{\imath}\right)=m\left(r^{\prime}(D), T_{\imath, \varepsilon}, *\right)$. Considering the limit $\varepsilon \rightarrow 0$ and taking (2.2) into account, we have

$$
m\left(r^{\prime}(D), T_{\varepsilon}, *\right)=\sum_{\imath=1}^{k} m\left(r^{\prime}(D), T_{\imath, \varepsilon}, *\right)=\sum_{\imath=1}^{k} m\left(r^{\prime}(D), \Delta_{\imath}, P_{\imath}\right)=\eta([g]) \cdot[D] .
$$

Combining this with (2.3), we complete the proof.

\section{Proof of Proposition 3}

We put

$$
T:=\left\{X_{0} X_{1} X_{2}=0\right\} \subset \boldsymbol{P}^{2} .
$$

Then the group $\left(\boldsymbol{G}_{m}\right)^{3} /$ (diagonal) acts on $\boldsymbol{P}^{2} \backslash T$ and on $\mathfrak{d}(S)$ compatibly by

$$
\boldsymbol{P}^{2} \backslash T \ni(a: b: c) \longmapsto(\lambda a: \mu b: \nu c) \quad \text { for }(\lambda, \mu, \nu) \in\left(\boldsymbol{G}_{m}\right)^{3} .
$$

This action on $\boldsymbol{P}^{2} \backslash T$ is transitive. Therefore we have $\mathrm{Bs}(\mathcal{D}(S)) \subset T$. In particular, the fixed components of $\mathfrak{b}(S)$ must be contained in $T$. On the other hand, the assumption implies that no lines in $T$ can be a fixed component of $\mathfrak{D}(S)$. Hence $\mathfrak{d}(S)$ does not have any fixed component.

We consider the restriction

$$
\Phi^{\prime}: \boldsymbol{P}^{2} \backslash T \longrightarrow \delta(S)^{`} \cong \boldsymbol{P}^{s} \quad(s+1=\operatorname{Card} S)
$$

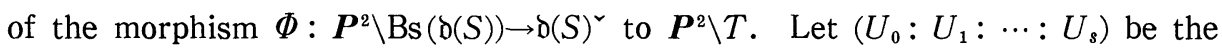
homogeneous coordinates of $\mathfrak{D}(S)^{\vee}$ dual to the basis $\left\{X_{0}^{i} X_{1}^{j} X_{2}^{k}\right\}_{(i, \jmath, k) \in S}$ of $\mathfrak{d}(S)$. We denote by $X_{0}^{i_{\nu}} X_{1}^{j_{\nu}} X_{2}^{k_{\nu}}$ the monomial corresponding to $U_{\nu}$. Then the image of $\Phi^{\prime}$ is contained in

$$
\boldsymbol{P}^{s} \backslash\left\{U_{0} U_{1} \cdots U_{s}=0\right\} .
$$

By changing the numbering if necessary, we may assume that the three vectors $\left(i_{0}, j_{0}, k_{0}\right),\left(i_{1}, j_{1}, k_{1}\right)$, and $\left(i_{2}, j_{2}, k_{2}\right)$ are linearly independent over $\boldsymbol{Q}$ because of the assumption. There exist a positive integer $N$ and integers $a_{\mu}, b_{\mu}, c_{\mu}(\mu=$ $0,1,2)$ such that

$$
\sum_{\mu=0}^{2} a_{\mu}\left(i_{\mu}, j_{\mu}, k_{\mu}\right)=(N, 0,0)
$$




$$
\begin{aligned}
& \sum_{\mu=0}^{2} b_{\mu}\left(i_{\mu}, j_{\mu}, k_{\mu}\right)=(0, N, 0), \\
& \sum_{\mu=0}^{2} c_{\mu}\left(i_{\mu}, j_{\mu}, k_{\mu}\right)=(0,0, N) .
\end{aligned}
$$

Let $\Psi$ be the composition of the morphisms

$$
\begin{aligned}
& \Psi: \boldsymbol{P}^{s} \backslash\left\{U_{0} \cdots U_{s}=0\right\} \longrightarrow \boldsymbol{P}^{2} \backslash\left\{U_{0} U_{1} U_{2}=0\right\} \longrightarrow \boldsymbol{P}^{2} \backslash\left\{V_{0} V_{1} V_{2}=0\right\} \\
& \left(U_{0}: \cdots: U_{s}\right) \longmapsto\left(U_{0}: U_{1}: U_{2}\right) \longmapsto\left(\prod_{\mu=0}^{2} U_{\mu}^{a}{ }_{\mu}: \prod_{\mu=0}^{2} U_{\mu}^{b_{\mu}}: \prod_{\mu=0}^{2} U_{\mu^{\mu}}^{c^{\mu}}\right) .
\end{aligned}
$$

The composition of $\Phi^{\prime}$ and $\Psi$ is given by

$$
\begin{gathered}
\boldsymbol{P}^{2} \backslash\left\{X_{0} X_{1} X_{2}=0\right\} \longrightarrow \boldsymbol{P}^{2} \backslash\left\{V_{0} V_{1} V_{2}=0\right\} \\
\left(X_{0}: X_{1}: X_{2}\right) \longmapsto\left(X_{0}^{N}: X_{1}^{N}: X_{2}^{N}\right) .
\end{gathered}
$$

Therefore it is finite and étale. Hence the image of $\Phi^{\prime}$ is of dimension 2 and no curves in $\boldsymbol{P}^{2} \backslash T$ are mapped to a point by $\Phi^{\prime}$. On the other hand, the assumption implies that no lines in $T$ are mapped to a point by $\Phi$. Thus the assumption (ii) in Proposition 1 holds. Using Propositions 1 and 2, we complete the proof.

\section{Other examples}

Example 3. Let $V \subset \boldsymbol{P}^{N}$ be a nonsingular projective variety of dimension $\geqq 2$. Suppose that $V$ is simply connected. Let $S \subset \boldsymbol{P}^{N}$ be a general hypersurface of degree $d$. Since $\pi_{2}(V) \cong H_{2}(V, \boldsymbol{Z}) \neq 0$, and the linear map $H_{2}(V, \boldsymbol{Z}) \rightarrow \boldsymbol{Z}$ induced from the intersection with $[V \cap S] \in H_{2 n-2}(V, Z)$ is non-trivial, Proposition 2 implies that $\pi_{1}(V \backslash S)$ is a finite cyclic group, and its order is in proportion to $d$.

Example 4. Let $V$ and $S$ be as in Example 3. If $V$ is a complete intersection, then $\pi_{1}(V \backslash S) \cong Z /(d)$. Indeed, by the generalized Lefschetz-Zariski Theorem due to Goresky-MacPherson ([2]), we have $\pi_{1}(H \cap(V \backslash S)) \cong \pi_{1}(V \backslash S)$ for a general hyperplane $H \subset \boldsymbol{P}^{N}$ if $\operatorname{dim} V \geqq 3$. Therefore, we may assume that $\operatorname{dim} V$ is large enough compared with its multi-degree. Then $V$ contains a line and its class generates $H_{2}(V, \boldsymbol{Z}) \cong \boldsymbol{Z}$. Since $S$ is general, this line intersects $S$ at distinct $d$ points transversely. Hence the cokernel of the boundary map $\pi_{2}(V)$ $\cong H_{2}(V, \boldsymbol{Z}) \rightarrow \boldsymbol{Z}$ is $\boldsymbol{Z} /(d)$.

\section{REFERENCES}

[1] A. Dimca, Singularities and Topology of Hypersurfaces, Springer-Verlag, Berlin, 1992.

[2] M. Goresky and R. MacPherson, Stratified Morse Theory, Springer-Verlag, Berlin, 1988. 
[3] M. Nori, Zariski's conjecture and related problems, Ann. Sci. École Norm. Sup. (4), 16 (1983), 305-344.

[4] I. Shimada, Fundamental groups of open algebraic varieties, to appear in Topology.

Department of Mathematics

FACUlty of Science

HOKKAIDO UNIVERSITY

SAPPORO, 060 JAPAN

e-mail : shimada@math.hokudai.ac.jp 